\title{
VIDEO CONTROLLING USING HAND GESTURE FOR DISABLED PEOPLE USING CONVEX HULL TECHNIQUE
}

\author{
Stella Nadar, Simran Nazareth, Kevin Paulson, Nilambari Narkar \\ Department of Computer Engineering, Xavier Institute of Engineering, Mumbai University, Mumbai, India
}

\begin{abstract}
The development of human-computer interaction (HCI) is a non-tangible way of communication between a human and a computer. Human hand gesture recognition (HGR) is one of the main topics in the area of computer science as an effective approach for HCI. Our project aims at developing a video player controlled by human hand gestures using the Convex Hull technique. This application uses the webcam to capture gestures by the user and perform basic operations such as play and pause. The gesture would serve as the direct command for operations such as play or pause the video based on the user's gestures onto the screen. The project is implemented using Python, OpenCV. We can use our hand gestures to control certain functions of a computer like playing or pausing a video, increasing and decreasing volume, etc.
\end{abstract}

Keywords - : HCI (Human Computer Interaction), Convex Hull, OpenCV, VLC controller, Webcam.

\section{INTRODUCTION}

Among many computer visions based interactive systems, designing hand gestures and facial expression-based Human-Computer-Interaction (HCI) systems remains to be a highly challenging task. Our main purpose is to find a nontangible way to interact with the computer. Our project aims at developing a video player controlled by human hand gestures. The gestures would serve as the direct command for operations such as play or pause the video. It uses simple gestures to control the video. So, people don't have to learn machine-like skills which are a burden most of the time, but against this people need only to recollect a group of gestures to control the video playback. In this paper, we have discussed a low system that uses hand gesture recognition technology to control the VLC media player.

\section{A. Aims and Objectives}

There are a lot of critical situations in the day-to-day lives of disabled people. We have come up with a small part of such life to make it easier using computer vision technology. In this paper, we have discussed a low system that uses hand gesture recognition technology to control the VLC media player. Among many computer visions based interactive systems, designing hand gestures and facial expressionbased HCI system retains to be a highly challenging task. Our main purpose is to find a nontangible way to interact with the computer.
Our project aims at modifying the existing video player controlled by human hand gestures by making use of Convex Hull and OpenCV.

Objectives:

1) To minimize the use of keyboard and mouse in computer.

2) To integrate gesture recognition features into any computer at a low cost.

3) To help in the development of a non-tangible way to interact with the video player.

\section{B. Scope of the Project}

The Hand Gesture recognition is moving at tremendous speed for the futuristic products and services and major companies are developing technology based on the hand gesture system and that includes companies like Microsoft, Samsung, Sony and it includes the devices like Laptop, Hand held devices, Professional and LED lights. The use and adoption will become more cost effective and cheaper. It's a brilliant feature turning data into features with mix of technology and Human wave. Smart phones have been experiencing enormous amount of Gesture Recognition Technology with look and views and working to manage the Smartphone in reading, viewing and that includes what we call touch less gestures. In the medical fields Hand Gesture may also be experienced in terms of Robotic Nurse and medical assistance. As the Technology is always revolving and changing the future is quiet unpredictable but we have 
to be certain the future of Gesture Recognition is here to stay with more and eventful and Life touching experiences.

\section{Existing System}

Using Convex Hull and Gestures for Human-Computer Interaction (HCI) may be a pretty recent field of investigation. In that respect, only a couple of articles are published on that topic. The existing system that we are looking at is a video player controlled by hand gesture movements and postures. Development of HumanComputer Interaction (HCI) is a non-tangible way of communication between a human and computer. Our project aims at developing a video player controlled by human hand gestures using the Convex Hull concept. This application uses the webcam to capture gestures from the user and perform basic operations.

\section{RELATED WORK}

As the aim was to use the Convex Hull concept to achieve the classification, Approach towards the necessity of Human-Machine Interaction using OpenCV along with image acquisition, motion detection, thresholding, contouring, segmenting hand region effectively from a real time video sequence. A video stream is just a collection of frames at a particular time. So if we are successful in extracting those frames then it would be the initial step for our objective. These frames then get processed under image acquisition techniques using OpenCV.

The developed model generates multiple channels of information from the input frames, and the final feature representation combines information from all channels. To further boost the performance, we propose regularizing the outputs with high-level features and combining the predictions of a variety of different models. We apply the developed Convex Hull concept code to recognize human actions in the real-world environment of the airport surveillance videos and they achieve superior performance in comparison to baseline methods.

Understanding an image and classifying it into different hand gestures using Convex Hull, where we predict a class for each image. These images output in the form of an input to a modified VLC system then can allow the system to perform commanded actions. Analysing this gesture is useful in the following ways - 1 . For a disabled individual who is unable to walk around in search of the system control devices (remote controller) or to press any keys on the system. 2. For an individual who is unable to understand the upgraded skill of the controller (how to operate the controller). 3. For quick and efficient action on the video running in an emergency.

\section{DESCRIPTION}

A. Analysis

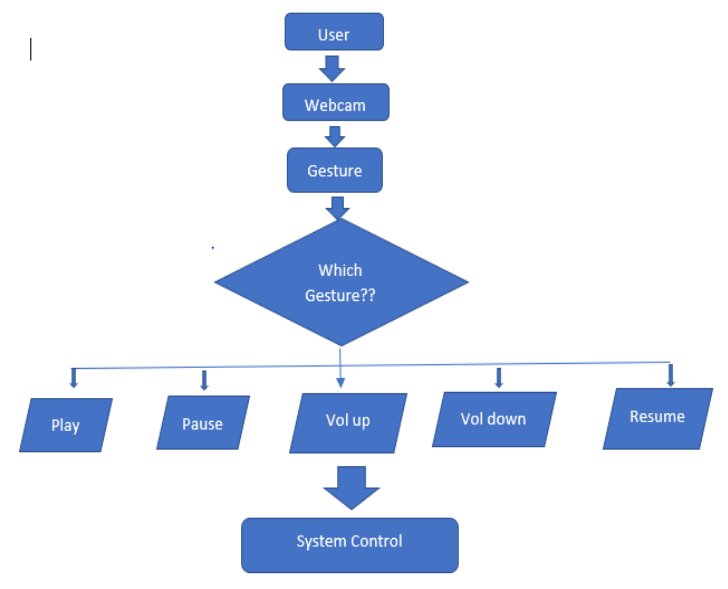

Fig. 1 Basic Architecture of the Project

Figure 1 is the basic structure that we plan to achieve where the simple human hand gestures will be considered as a control input for the video player to perform actions based on the appropriate mapping of the gestures along with their meaningful control actions (play, pause, volume up, volume down, resume).

\section{B. Feasibility Study}

1) Economic Feasibility: The cost of the project depends upon the accuracy of image capturing and pre-processing of those images using different techniques and finally the efficiency of the Convex Hull concept. Hence, by costbenefit analysis we can conclude that benefit to cost ratio is not much high.

2) Technical Feasibility: As mentioned earlier, the task of coding and debugging was made easier by using Convex Hull for the detection. As we use only a webcam to capture images, much GUI is not required, the app construction coding was also not a very difficult task. As every segment will be coded individually and separately, the risk assessment for each segment is easy. 
3) Operational Feasibility: The user just has to upload a live video using a webcam. The image just has to be of high quality which can easily be captured from the webcam and processed. Hence, the controls are simple and basic. The efficiency of the project is based upon few factors, namely, the concept and its individual accuracy, the front end and its latency, the inter-connectivity. All these components can again be controlled individually and hence accuracy of the project as a whole can be easily controlled.

\section{Design}

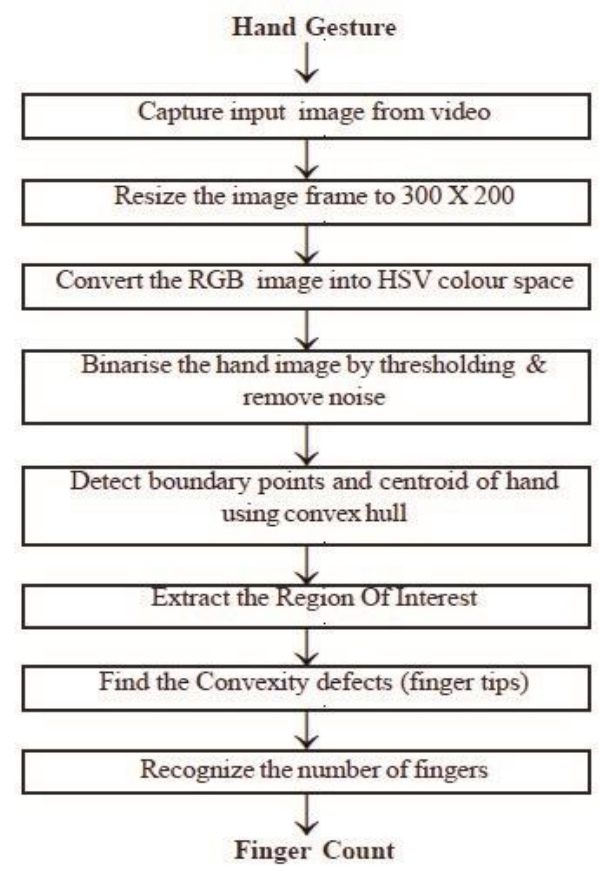

Fig. 2 Flowchart of the Image Detection

The steps for Image Processing is been represented using Figure 2 which consists of eight steps such as capturing, resizing, converting, thresholding, contouring, extracting, detecting then finally recognizing.

\section{IMPLEMENTATION}

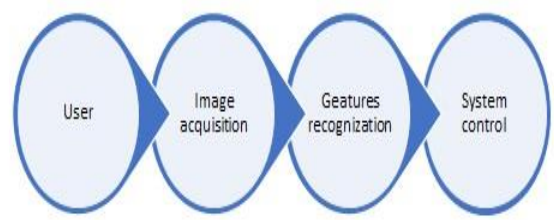

The design for the proposed system is shown in Figure 3 The images and their masked labels are in .png format. The images are being captured from the live video stream and these images are further processed to achieve efficiency of the detection stage. In the detection stage, initially, contours are identified for the images and then these contours are used for the creation of the Convex Hull around the hand region. Then these image outputs are passed as control commands to the VLC media player to perform the associated action.

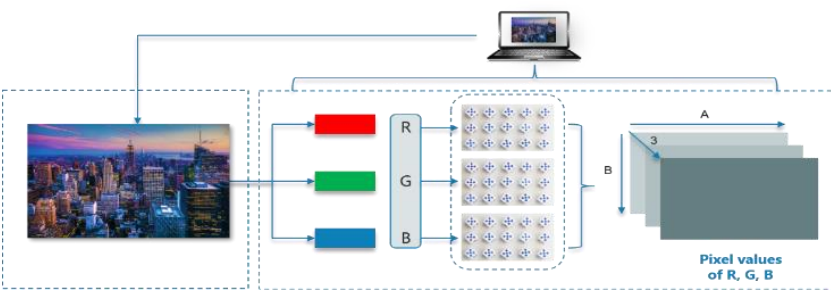

Fig. 4 Computer understanding Image

As from figure 4 it is clear that, using three dimensions matrix pixel values (RGB values) for all the images in it crucial to distinguish between these images. Semantic Segmentation technique where the objective is to find different regions in an image and tag its corresponding labels, for this first step includes segmenting only the hand region from the live video stream and identify different possible gestures. The convex hull cluster is of peaks that cover the region of the hand. Here, we must clear the principle of the convex set, which means all lines between any 2 points within the hull are entirely within it. After determining the gesture, the specific functioning is performed. The method of recognizing the movement is a dynamic process. After operating the specific command from the gesture, go back to the initial step to accept other images to be processed, and so on.

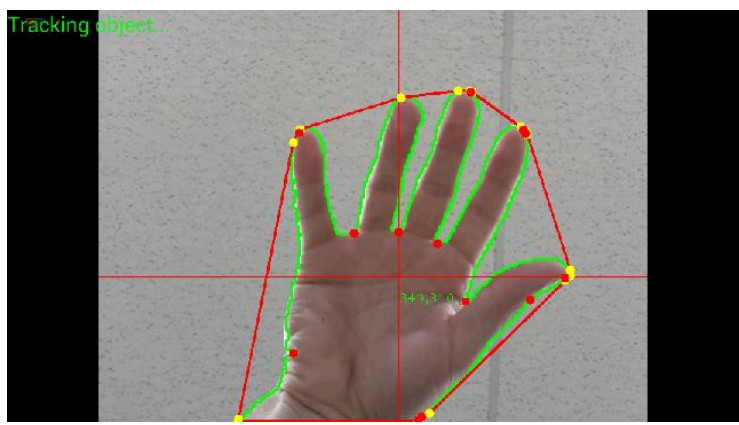

Fig. 5 Contours Convex Hull boundary 
After this the gesture pictures were segregated into labelled folders as play, pause, volume up, volume down and resume appropriately. Exploratory Image details Analysis is an approach for analysing details related to images to summarize their main characteristics, often with visual methods. Much complex visualization can be achieved with matplotlib and usually, there is no need to import other libraries. This is used for gaining a better understanding of data aspects like:

-main features of data

-variables and relationships that hold between them -identifying which variables are important for our problem Next we plan to input this processed images as input into the VLC media player and perform the necessary actions.

\section{RESULTS}

Depending on the contour defects and making use of OpenCV techniques the gestures are identified and classified into different gestures and there are visual threshold and color representations in the result. During the live video, streaming frames are extracted, processed, and converted into proper images along with region segmentation then these image outputs are supplied as input into the VLC video player to perform the associated action command related to VLC. Here we have implemented and restricted our project only for 4 gestures further scope would be to increase the number of gesture recognition and commands for the VLC video player.

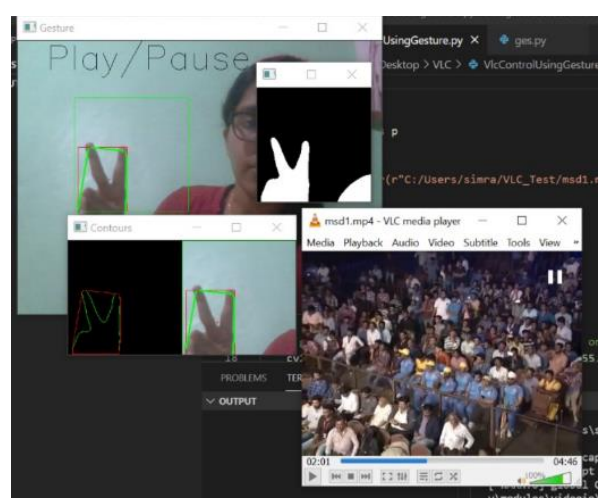

Fig. 6 Play Action using Hand's Gesture

The system is successful in detecting the hand gesture for play and recognizing the action to be performed, so the corresponding action of playing the video is functioned.

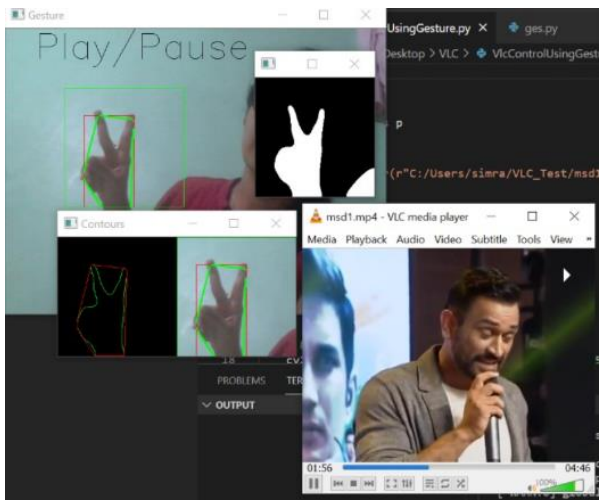

Fig. 7 Pause Action using Hand's Gesture

The system is successful in detecting the hand gesture for pause and recognizing the action to be performed, so the corresponding action of pausing the video is functioned.

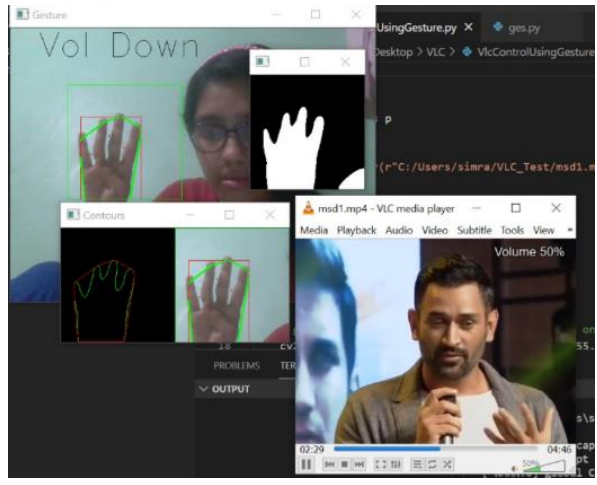

Fig. 8 Volume Down Action using Hand's Gesture

The system is successful in detecting the hand gesture for Volume down and recognizing the action to be performed, so the corresponding action of decreasing the volume for the video is functioned.

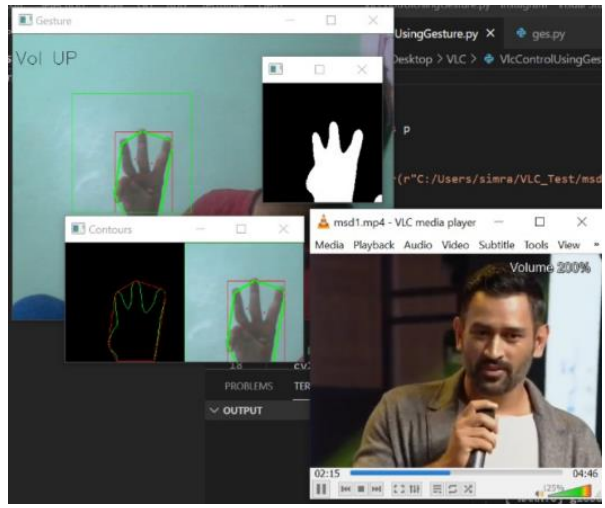

Fig. 9 Volume Up Action using Hand's Gesture 
The system is successful in detecting the hand gesture for Volume up and recognizing the action to be performed, so the corresponding action of increasing the volume for the video is functioned.

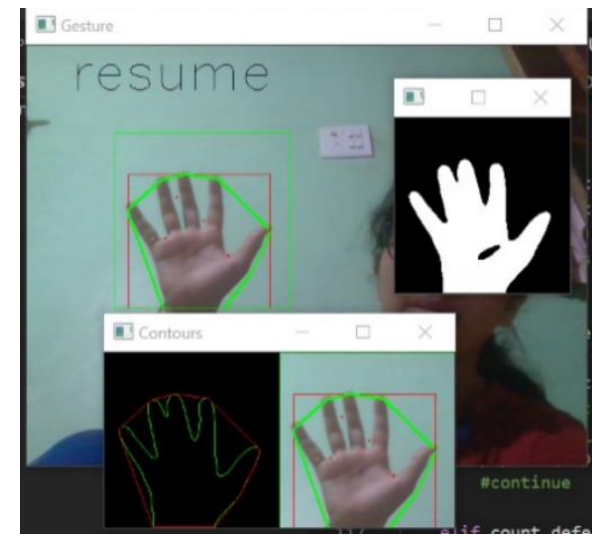

Fig. 10 Resume Action using Hand's Gesture

The system is successful in detecting the hand gesture for Resume and recognizing the action to be performed, so the corresponding action of resuming the video is functioned.

\section{PLANS FOR FUTURE}

As per our plans, we have completed our project implementation.

Next, we may aim to come with more gestures and their associated actions to be performed for building a completely modified VLC media player. A considerable amount of research yet needs to be done in this field as we need to take latency into consideration. The final aim is to reduce the project response time in real life as even a second delay could cause different control to be functioned.

\section{CONCLUSION}

The gesture would serve as the direct command for operations such as play or pause the video based on the user's gestures onto the screen. So people don't have to learn machine-like skills which are a burden most of the time, but by contrast, people need only to remember a set of gestures to control the video playback. The Hand Gesture recognition is moving at tremendous speed for the futuristic products and services and major companies are developing a technology based on the hand gesture system and that includes companies like Microsoft, Samsung, Sony and it includes the devices like Laptop, Handheld devices,
Professional and LED lights. Smartphones have been experiencing an enormous amount of Gesture Recognition Technology with look and views and working to manage the Smartphone in reading, viewing and that includes what we call touch less gestures.

\section{REFERENCES}

[1] Stella Nadar, Simran Nazereth, Kevin Paulson, Nilambari Narkar, "Video Controller using Hand Gesture for Disabled People” In: IJTRET(2021).

[2] Soumik Mondal Gaurav Sharma. "A Dynamic Hand Gesture Recognition System for Controlling VLC Media Player". In: International Conference on Advances in Technology and Engineering (ICATE). 2013.

[3] Swapna Agarwal, Saiyed Umer. "MP-FEG: Media Player controlled by Facial Expressions and Gestures". In: Fifth IEEE National Conference on Computer Vision, Pattern Recognition, Image Processing and Graphics (2015).

[4] Murthy G., Jadon R. "A review of vision based hand gestures recognition.” In: International Journal of Information Technology and Knowledge Management. (2009). 